\title{
Publisher Correction: Critical Southern Ocean climate model biases traced to atmospheric model cloud errors
}

Patrick Hyder ${ }^{1}$, John M. Edwards ${ }^{1}$, Richard P. Allan (1D 2,3, Helene T. Hewitt ${ }^{1}$, Thomas J. Bracegirdle ${ }^{4}$, Jonathan M. Gregory ${ }^{1,5}$, Richard A. Wood ${ }^{1}$, Andrew J.S. Meijers ${ }^{4}$, Jane Mulcahy ${ }^{1}$, Paul Field ${ }^{1,6}$, Kalli Furtado ${ }^{1}$, Alejandro Bodas-Salcedo ${ }^{1}$, Keith D. Williams' ${ }^{1}$, Dan Copsey ${ }^{1}$, Simon A. Josey ${ }^{7}$, Chunlei Liu (D ${ }^{2,3}$, Chris D. Roberts ${ }^{1}$, Claudio Sanchez ${ }^{1}$, Jeff Ridley ${ }^{1}$, Livia Thorpe ${ }^{1}$, Steven C. Hardiman', Michael Mayer ${ }^{8}$, David I. Berry ${ }^{7} \&$ Stephen E. Belcher ${ }^{1}$

Correction to: Nature Communications; https://doi.org/10.1038/s41467-018-05634-2; published online 11 September 2018

In the original HTML version of this Article, ref. 12 was incorrectly cited in the first sentence of the first paragraph of the Introduction. The correct citation is ref. 2. This has now been corrected in the HTML version of the Article; the PDF version was correct at the time of publication.

Published online: 02 October 2018 ceproduction in any medium or format, as long as you give appropriate credit to the original author(s) and the source, provide a link to the Creative Commons license, and indicate if changes were made. The images or other third party material in this article are included in the article's Creative Commons license, unless indicated otherwise in a credit line to the material. If material is not included in the article's Creative Commons license and your intended use is not permitted by statutory regulation or exceeds the permitted use, you will need to obtain permission directly from the copyright holder. To view a copy of this license, visit http://creativecommons.org/licenses/by/4.0/.

(C) The Author(s) 2018

\footnotetext{
${ }^{1}$ Met Office Hadley Centre, FitzRoy Road, Exeter, UK. ${ }^{2}$ Department of Meteorology, University of Reading, PO Box 243Whiteknights Campus Earley Gate, Reading RG6 6BB, UK. ${ }^{3}$ National Centre for Earth Observations (NCEO), University of Reading, PO Box 243 Whiteknights Campus Earley Gate, Reading RG6 6BB, UK. ${ }^{4}$ British Antarctic Survey, High Cross, Madingley Road, Cambridge CB3 OET, UK. ${ }^{5}$ National Centre for Atmospheric Sciences (NCAS), University of Reading, PO Box 243Whiteknights Campus Earley Gate, Reading RG6 6BB, UK. ${ }^{6}$ School of Earth and Environment, University of Leeds, Leeds LS2 9JT, UK. ${ }^{7}$ National Oceanography Centre, European Way, Southampton SO14 3ZH, UK. ${ }^{8}$ Department of Meteorology and Geophysics, University of Vienna, Althanstraße 14, Vienna 1090, Austria. Correspondence and requests for materials should be addressed to P.H. (email: patrick.hyder@metoffice.gov.uk)
} 